\title{
A Study of the Sources and Information Collection and Metric Model of Software Requirements Change
}

\author{
Yuqing Yan ${ }^{1, a}$, Weijun Sun ${ }^{2, b}$, Zhong Ming ${ }^{3, c}$ and Zhenhua Zhang ${ }^{4, d^{*}}$ \\ ${ }^{1}$ School of Finance, Guangdong University of Foreign Studies, Guangzhou 510006, China \\ 2 School of Automation, Guangdong University of Technology, Guangzhou 510006, China \\ ${ }^{3}$ College of Computer and Software, Shenzhen University, Shenzhen 518060, China \\ ${ }^{4}$ School of International Trade and Economics, Guangdong University of Foreign Studies, 510006, \\ China \\ ayyq@gdufs.edu.cn, bgdutswj@gdut.edu.cn, c56238305@qq.com, d63221506@qq.com
}

Keywords: Requirements change; Origin; Request; Information collection; Metric.

\begin{abstract}
The sources of requirements change should firstly be identified in the process of managing requirements, while the collection of information and the measurement of change activities should be conducted by methodology. This paper firstly reviewed the state of the arts of identifying and classifying requirements change sources in literature, pointed out the shortcomings of the present research. A new taxonomy for hierarchically identifying and classifying the sources of change were proposed based on four principles proposed. Thus, according to the formal steps of change management, three forms of collecting change information were suggested. The way to use these collected data to measure the process of requirements change was discussed. The results of this paper will provide some methods to carry out future empirical research concerning requirements change in industries.
\end{abstract}

\author{
软件需求变化信息采集和度量框架研究 \\ 严玉清 ${ }^{1, a}$ ，孙为军 ${ }^{2, b}$ ，明仲 ${ }^{3, \mathrm{c}}$ ，张振华 ${ }^{4, \mathrm{~d}}$ \\ 1 广东外语外贸大学金融学院, 广州, 广东, 510006, 中国 \\ 2 广东工业大学自动化学院, 广州, 广东, 510006, 中国 \\ 3 深圳大学计算机与软件学院, 深圳, 广东, 518060, 中国 \\ 4 广东外语外贸大学经贸学院, 广州, 广东, 510006, 中国
}

ayyq@gdufs.edu.cn, bgdutswj@gdut.edu.cn, c56238305@qq.com, d63221506@qq.com

关键词: 需求变化; 来源; 请求; 信息采集; 度量

中文摘要. 需求变化管理首先要识别变化来源, 变化信息的收集和变化活动的度量需要建立一定的方 法指导。本文首先简要回顾在需求变化来源的识别和分类方面的研究现状和特点, 针对当前研究的不 足, 提出了识别和分类变化来源的四原则, 在此基础上, 建立了一个新的需求变化来源分类模型。根 据变化管理步骤, 构建了三个变化信息收集表单, 探讨了如何利用这些分段收集到的数据去度量需求 变化风险和其它反映需求分析效率相关的量。本文的结果能够为需求变化的实证研究以及定量研究提 供方法指导。 


\section{1. 引言}

需求变化是软件开发和维护面临的主要挑战。上世纪八十年代开始, 学术界对需求变化 问题广泛关注。其中, 需求变化的来源的识别和分类成为关注的焦点 ${ }^{[1]}$ 。构建可信的软件需 求, 需要识别来自软件系统内部和外部的各种威胁, 这些威胁形成需求变化的来源 $[2-3]$ 。研究 变化来源有助于理解需求及变化的本质, 建立预测需求变化的方法, 有效管理需求变化和软 件风险。

\section{2. 需求变化的来源及其分类}

\section{1 当前研究现状}

上世纪90年代初, Harker等 ${ }^{[1]}$ 研究用户和组织需求的易变性, 根据易变需求的类型定义相 应的变化来源。在此基础上, Kotonya等 ${ }^{[4]}$ 研究需求变化管理的步骤及需求变化来源的分类。 我们可以看到, 动态变化的环境、缺乏用户的参与、开发人员经验不足都会引发需求变化 ${ }^{[3]}$ 。 表2.1根据当前研究现状，从几方面简要概括了需求变化来源在分类方面的情况。

表2.1 需求变化来源、原因及其分类

\begin{tabular}{|c|c|c|c|c|c|}
\hline 涉及阶段 & 关注焦点 & 类别数 & 来源路径 & 研究方法 & 文献出处 \\
\hline 需求过程 & 用户和组织需求的变化性 & 5 & 无 & 文献分析 & {$[1]$} \\
\hline 开发过程 & 需求变化来源及变化类型 & 4 & 无 & 实证研究 & {$[5]$} \\
\hline 开发过程 & 需求变化来源及影响分析 & 10 & 可发现两层 & 实证分析 & {$[6]$} \\
\hline 开发过程 & 需求变化来源分析、实证研究 & 5 & 有: 三层 & 实证分析 & {$[7]$} \\
\hline 开发过程 & 需求变化原因分析及其分类 & 3 & 部分 & 文献分析 & {$[8]$} \\
\hline 需求过程 & 变化原因分析及预防策略 & 4 & 有: 三层 & 文献分析 & {$[2]$} \\
\hline 开发过程 & 管理变化需求 & 8 & 不明显 & 问卷调查 & {$[9]$} \\
\hline
\end{tabular}

\section{2新的需求变化来源分类模型}

需求变化是一种复杂的现象。它的来源需要得到彻底研究。为了建立需求变化来源识别 和分类的方法学, 我们提出了四个原则 ${ }^{[3]}$ : (1) 全局性(Holistic): 在软件生命周期中, 许多因 素会诱发软件风险, 这些因素同时也诱发需求变化。因此, 在挖掘变化因素时, 要从整体角 度加以考虑; (2) 相关性(Correlative): 许多因素相互间存在着联系, 因此, 在挖掘变化因素 时, 要考虑因素之间的相关性; (3) 强内聚性(Strong Cohesive): 要把关系紧密的因素划分在 一个类别中; (4) 弱耦合性(Weak Coupling): 由于因素之间关系的复杂性, 不同类别的因素之 间或多或少存在着一定的联 系, 但要尽可能减少这些联系 的紧密性。

根据这四个原则, 我们建 立了如图2.1所示的层次化需 求变化来源模型。从该图可见, 在软件生命周期中, 可从两大 阶段去挖掘需求变化来源, 而 且这两个阶段的来源之间存在 着交集。这一交集通常不会为 空集, 且会动态变化。这取决

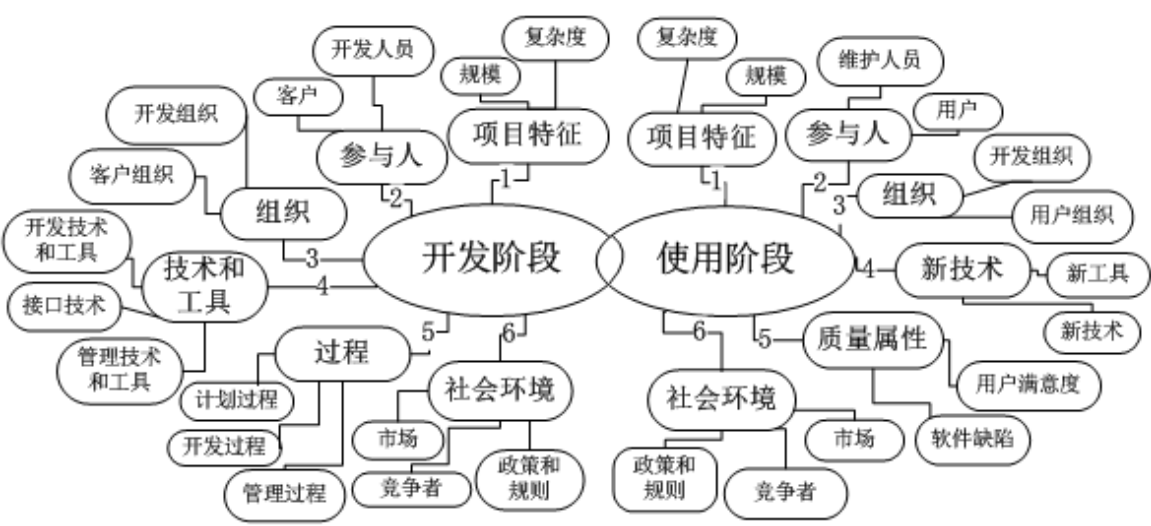

图2.1 需求变化的层次来源结构 于所交付产品的质量、组织和市场的稳定性以及软件的使用环境。如果软件质量不高或市场 中存在强有力的竞争性软件或组织不稳定，那么这个交集将会增大。 
这个模型的特点是把软件风险和需求变化因素的识别放在同一个视角下, 能够看清需求 变化的本质, 而且建立了清晰的路径去查找各种变化因素, 使变化的管理更加有针对性。

\section{3 需求变化信息采集和变化活动过程度量框架}

实证法是软件工程领域重要的研究方法, 同时也是研究需求变化的重要方法, 但目前关 于需求变化信息的收集缺乏有效的方法指导。

\section{1 当前研究现状}

IBM 的 Stark 等 ${ }^{[5]}$ 早在 1998 年就对航空软件维护过程中的需求变化影响进行了实证研究。 他们收集的数据包括: 需求的类型、计划和实际花费在每一需求上的成本、需求变化的类型、 提请需求变化的组织类型、需求变化的频率。澳洲悉尼科技大学的 Nurmuliani 等 ${ }^{[6]}$ 在实证研 究需求变化及其对成本的影响时, 收集变化请求的数据有: 变化的属性(理由、类型、来源)、 变化的成本。美国软件工程专家 Wiegers ${ }^{[10]}$ 建立了一个软件风险信息跟踪模板, 包括十项条目: ID 号、风险确认日期、风险撤消日期、以 “条件-结果” 的形式描述的风险、风险可能的危害 影响、风险危害值、降低风险的计划、解决风险的负责人、实施风险管理的截止日期。上世 纪九十年代中叶, 英国著名学者 Ian Sommerville 曾经建立了一个需求变化请求表单 ${ }^{[11]}$, 包括 22 个条目, 其中关键条目有: 变化请求者、待变化需求的内容、变化分析者、受影响的构件、 与之相关的构件、变化难易程度评估、变化优先级、变化成本估算。尽管如此, 关于需求变 化活动信息的收集仍然缺乏方法指导, 缺乏灵活和方便使用的模板。这里, 基于前人的研究, 结合我们的研究经验, 按照需求变化管理步骤 ${ }^{[11]}$, 从方法学角度提出三个变化信息采集表单。

\section{2 需求变化信息采集表单}

需求变化信息的收集, 可以从建立了第一个需求基线之后开始 ${ }^{[10]}$ 。每一轮变化过程完成, 都会产生新的需求基线。为了提高信息收集效率，有必要分阶段、分步骤来实施信息的收集。

3.2.1 变化请求表单

变化的类型通常有三种 ${ }^{[5]}$ : 增加(add)、删除(delete)和修改(modify)。需求增加往往是因为 项目范围扩大。当需求获取不完整, 需求理解和表达不正确或需求圥余等需求问题发生时, 变化请求便会发生。根据变化管理步骤, 必须有一个正式提交变化请求的过程。请求表单可 设计如表 3.1 所示(表单 I )。其中，(1) “项目名称” 指当前项目的名称; (2) “ID 号” 是提请 变化的需求编号(每一编号对应具体的需求, 通常用自然语言表达)(新增需求可编制临时编 号); (3) “需求类型” 通常分为功能需求和非功能需求, 也可采用更加具体、细致的分类 ${ }^{[11]}$; (4) “请求内容” 是提请变化的建议和要求, 包括要采取的行动和措施, 例如: “通话记录的 查询方法要补充详细的文字说明”; (5) “提交者” 是提出需求变化的人或部门; (6) “日期” 是请求提出的日期; (7) “理由” 简要说明提出此请求的缘由; (8) “变化类型” 是对

表3.1 需求变更请求信息数据项

\begin{tabular}{|c|c|c|c|c|c|c|c|c|c|c|}
\hline 项目 & ID & 需求 & 请求 & 提交 & 日 & 理 & 变更 & 变更 & 阶 & 备 \\
名称 & 号 & 类型 & 内容 & 者 & 期 & 由 & 类型 & 来源 & 段 & 注 \\
\hline
\end{tabular}

需求实施的操作类型; (9) “变化来源” 可在现有的来源分类结构中找出适用的方法, 最好 能够提供确定变化来源路径的方法(例如图 2.1), 以明确问题发生的来龙去脉，以便后续的过 程管理更有针对性; (10) “阶段” 是该变化请求具体处于哪个开发阶段。明确具体阶段也是 使后续开发过程管理更有针对性的做法; (10) “备注” 可以包括一些必要的, 但在此表单中 没有提到的信息。例如, 某些变化请求被多次提出, 鉴于成本、优先级等的考虑, 在评审时 没有通过。通过这些信息的收集, 可以了解所提出的需求变化请求的分布情况, 有助于了解 需求获取和分析的效率，使后续的开发和管理更加有针对性。

3.2 .2 变化请求评审与实施表单

在收集了需求变化请求信息之后, 需要对请求数据进行分析、评价, 为变化管理组织的 决策提供依据。分析、评价和决策信息可用表 3.2(表单 II)加以记录。 
表3.2 评审需求变化请求信息数据项

\begin{tabular}{|c|c|c|c|c|c|c|c|c|c|c|c|c|c|c|c|}
\hline 项目 & ID & 相关 & 影响 & 影响 & 影响 & 变化 & 优先 & 风险 & 评审 & 评审 & 决策 & 决策 & 决策 & 决策 \\
名称 & 号 & 构件 & 构件 & 成本 & 进度 & 难度 & 级 & 估计 & 者 & 日期 & 结果 & $\begin{array}{c}\text { 理由 } \\
\text { 者 }\end{array}$ & 日期 & 注 \\
\hline
\end{tabular}

其中，(1) “项目名称” 同表 3.1；(2)“ID 号”同表 3.1；(3)“相关构件”是指与该提请 变化的需求相关的需求或设计模块或代码模块; (4) “影响构件” 是受该需求变化影响的其它 软件构件。要发现这些构件, 需要借助需求跟踪方法; (5) “影响成本” 是该变化产生的成本; (6) “影响进度” 是该变化可能对开发进度的影响; (7) “变化难度” 是该变化的难易程度, 级 别通常为: 高、中、低; (8) “优先级” 是这一需求变化的优先级别, 通常为: 高、中、低; (9) “风险估计” 是该需求变化可能会对开发过程和软件质量产生的风险影响估计。这一估计 可以用文字来描述也可以用数值来表达; (10) “评审者” 即参加评审的人员；(11) “评审日期” 即进行评审的日期; (12) “决策结果” 为通过、不通过或待定; (13) “决策理由” 是对决策结 果进行的适当文字说明；(14）“决策者” 是参加决策的人员；(15) “决策日期” 是决策完成的 日期; (16) “备注” 可以对在此表单中未列出的内容的补充说明, 例如实现这一变化可能对 软件维护难度、可靠性有影响，这种影响需要加以说明。

需求变化请求的评审过程是对实现这一变化请求的风险估算的过程。表 3.2 中，从 “相 关构件”到 “风险估计”，中间这七个数据项属于需求变化风险影响评估的内容。

可以利用表 3.2 所提供的数据进行需求变化影响分析、成本估算和进度分析, 有助于加 深开发人员对需求的理解, 转个角度去认识和思考需求获取和分析过程。

在对需求变化请求作出决策之后, 需求变化实现即可开始。在变化实施之后, 需要对结 果和效果进行复查。在这一过程中, 需要记录的相关信息可使用表 3.3(表单III)的数据项。

表 3.3 需求变化实施信息数据项

\begin{tabular}{|c|c|c|c|c|c|c|c|c|}
\hline 项目 & ID & 结果 & 实施 & 花费 & 完成 & 复查 & 复查 & 备 \\
名称 & 号 & 需求 & 者 & 时间 & 日期 & 结果 & 者 & 注 \\
\hline
\end{tabular}

其中，(1) “项目名称” 同前述; (2) “ID 号” 是实现之后新需求的编号; (3) “结果需求” 是实现之后的新需求; (4) “实施者” 即实现这一需求变化的工程师; (5) “花费时间” 是实施 变化实际所花的时间；(6) “完成日期” 即完成这一变化的具体日期；(7) “复查结果” 即对实 施的变化效果进行检验的结果，可以是通过或不通过，对于不通过的，可用文字记录下问题 所在; (8) “复查者” 即进行复查的工程师; (9) “备注” 可以是对在此表单中未列出的数据项 的记录，例如可以记录实施、复查过程中发现的问题或解决问题的方法。

\section{3需求变化活动过程度量框架}

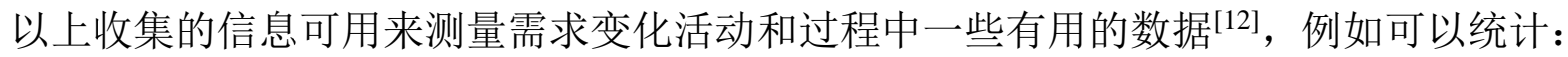
(1)接收、未作决定和已结束处理的变化请求的数量; (2)提出请求的需求类型、需求变化的类 型及其累计数量; (3)变化请求者的提议数目; (4) 已确定需求基线后, 需求变化请求和需求变 化的数目; (5)处理请求(变化请求的收集、分析、评价、决策)和实施变化所投入的总工作量(通 常计入成本中); (6)正确实施变化所经过的变化过程的反复次数 (有些时候需求变化实现不正 确, 或引起其它错误需要再次修正)。除了这六方面数量, 还可以进行: 7需求变化来源路径 数目统计; 8需求变化影响分析 ${ }^{[2]}$; 9观察、了解需求变化过程从请求开始至变化完成整个 过程的演化特征 ${ }^{[2,12]}$; 10总结易变需求的类型及其变化后的累计数量; (11)评估需求获取和分 析效率、开发人员的经验和开发效率以及 用户的参与积极性 ${ }^{[2,12]}$ 。

以上度量内容可分别在三个信息采 集表完成之后进行，具体情况如图 3.1 所 示。该图表明了需求变化过程与需求获取 和分析过程是交织在一起的循环往复的 过程。在确立了需求基线后，可使用表单

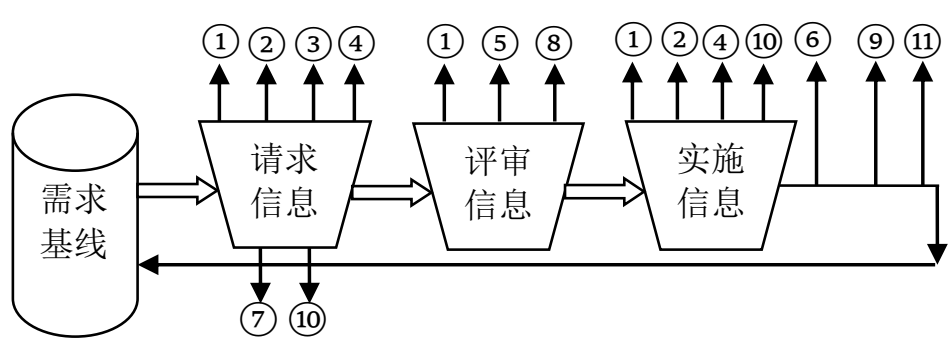

图3.1 需求变化信息采集及变化活动测量 
I 记录变化请求，获得的数据可以度量(1)、(2)、(3)、(4)、(7)和(10); 在评审信息(表单 II) 收集 完成之后, 可用来度量(1)、(5)和(8); 表单III的数据可用来度量(1)、(2)、(4)和(10。在变化过程 从请求到实现完成之后，会产生新的需求基线。同时，可利用前面收集到的数据来度量 (6)、

(9)和(11)。

\section{4 结束语}

对于软件可信性而言，涉及许多研究内容。例如：软件故障检测、基于测量的可信性评 估技术等等。本文所进行的研究属于基于测量的可信性评估技术的基础性研究，旨在提供一 个能更加仔细观察需求变化、理解其本质, 从多维度描述和度量需求变化活动及其风险影响 的实证方法，为理论和实践之间的沟通提供一个基础平台及起到一个抛砖引玉的作用。

\section{致谢}

本文为广东省自然科学基金项目 “序信息系统三支决策理论及其在软件外包项目全过程 风险管理中的应用研究”(No.2014A030313575), “基于风险微观视角及全球价值链的软件外包 产业转型升级战略路径研究” (2014A030313575), “技术服务外包产业发展升级与政府支持系 统研究-以广东省软件外包产业为例” (2015070704051), “中国公共数据库质量控制的多粒度 优化方法研究” (2016537)的阶段性研究成果之一。

\section{References}

[1] Harker S. D. P. and Eason K. D., Dobson J. E. The change and evolution of requirements as a challenge to the practice of software engineering[C]. Proceedings of the IEEE International Symposium on Requirements Engineering (RE 93), pp. 266-272, San Diego, California, 1993. USA, IEEE Computer Society Press.

[2] YAN Yuqing, SUN Weijun, WU Zhihui, ZHANG Zhenhua, MING Zhong. The Causes of Software Requirements Change and the Prevention Strategies [J]. Journal of Management Observer. 2016(02): 105-109. (in Chinese)

[3] Yu-Qing YAN, Zhen-Hua ZHANG. A method to connect the sources of software risk and requirements change[C]. 2016 International Conference on Management, Economics and Social Development (ICMESD 2016). Guilin, Guangxi, China, 2016: 1179-1185.

[4] Gerald Kotonya and Ian Sommerville. Requirements engineering-processes and techniques[M]. John Wiley \& Sons. 1997.

[5] Stark G, Oman P, Skillicorn A, et al. An examination of the effects of requirements changes on software releases[J]. Crosstalk the Journal of Defence Software Engineering, 1998, 11(5):11--16.

[6] N Nurmuliani, Didar Zowghi, Susan P. Williams. Requirements volatility and Its impact on change effort: evidence-based research in software development projects[C]. AWRE 2006 Adelaide, Australia, 2006:1-10.

[7] S. McGee and D. Greer. Software requirements change taxonomy: evaluation by case study[C]. 2011 IEEE 19th International Requirements Engineering Conference. pp.25-34.

[8] Bano, M., Imtiaz, S., Ikram, N., Niazi, M. \& Usman, M. Causes of requirement change-a systematic literature review[C]. in 'Proc. of Evaluation and Assessment in Software Engineering'. The institute of Engineering and Technology, 2012, pp. 22-31.

[9] Janes A, Remencius T, Sillitti A, et al. Managing changes in requirements: an empirical investigation[J]. Journal of Software: Evolution and Process, 2013, 25(12): 1273-1283. 
[10]Karl E. Wiegers. Software Requirements(2nd Edition)[M]. Microsoft Press, 2003.

[11]Ian Sommerville. Software engineering[M]. 9th Edition. Pearson Education Asia Limited and China Machine Press. 2011, 686.

[12] Yan Yuqing, Li Shixian, Sun Weijun, Huang Changqin. The Study of Requirements Evolution Based on Queuing Theory[J].Computer Science, 2012,39(5).pp:106-109. (in Chinese) 